\title{
Stem cell-derived erythrocytes as upcoming players in blood transfusion
}

\author{
A. R. Migliaccio ${ }^{1,2}$ \\ ${ }^{1}$ Department of Hematology, Oncology and Molecular Medicine, Istituto Superiore Sanità, Rome, \\ Italy \\ ${ }^{2}$ Tisch Cancer Institute, Mount Sinai School of Medicine, New York, NY, USA
}

\begin{abstract}
Background-Blood transfusion is current standard-of-care for genetic forms of anemia that would be otherwise lethal and allows implementation of aggressive cytotoxic/surgical therapies developed for numerous types of cancer. In developed countries the blood supply is adequate and sporadically even in excess. However, difficulties exist in finding blood with rare phenotypes to treat alloimmunized patients and the progressive ageing of the human population predicts that blood will become scarce by 2050. These considerations establish the need for the development of techniques to generate cultured red blood cell (cRBCs) as transfusion products.
\end{abstract}

Materials and Methods-Recent progress in cell culture techniques is revolutionizing organ replacement therapies. Two new disciplines, cell therapy and tissue engineering, have been developed to generate in vitro therapeutic products for a variety of applications ranging from skin grafts to organ-function repairs. It is currently believed that these advances will eventually allow ex-vivo production of various cell types in numbers so great that, in the case of red cells, would be clinically adequate for transfusion.

Results-Proof-of-principle in animal models indicate that cRBCs generated from murine embryonic stem cells protect mice from lethal anemia. Conditions to generate small amounts of clinical grade cRBCs have been established and the first-in-man administration of autologous cRBCs perfomed. The results of this trial indicate that cRBCs survive in vivo at least as long as their natural counterpart.

Discussion-These ground-breaking reports have raised great excitement for clinical evaluation of $c R B C s$ for transfusion. However, skepticism still persist that production of cRBCs in numbers sufficient for transfusion will ever be possible. This paper will discuss diagnostic and clinical goals pursuable with numbers of cRBCs that may be generated with current technology.

Conclusion-We are confident that development of relevant clinical goals achievable with current technologies will not only improve clinical care in transfusion medicine but will also foster

"Correspondence: Anna Rita Migliaccio, Tisch Cancer Center, Mount Sinai School of Medicine, One Gustave L. Levy Place, Box 1079, New York, NY 10029, USA annarita.migliaccio@mssm.edu.

Disclosure

The author has no conflicts of interest to declare. 
studies to overcome scientific and technical barriers that render transfusion with cRBCs of the general population impractical today.

\section{Keywords}

adult haematopoietic stem cells; anaemia; cord blood; embryonic stem cells; erythropoiesis; induced pluripotent stem cells

\section{Introduction - needs for alternative transfusion products}

Blood transfusion is an essential part of modern patient care and assurance that the blood supply is quantitatively and qualitatively adequate is both a national and international priority. The blood supply of industrialized countries is adequate overall [1-6]. Data from the World Health Organization indicate that over 93 million donations are made every year [7] and data from the US Department of Health and Human Services indicate that in the USA the number of units collected exceeded that of transfusions performed by $13 \%$ in 2008 [2]. However, with the identification of new infectious diseases transmittable by blood and the discovery of adverse events associated with transfusion of older units, the definition of blood quality is a moving target that may affect the blood supply [8].

Globalization of the world economy with migrations of populations to different geographical areas may also reduce blood supply: it exposes individuals to additional transfusion transmitted diseases, reducing the pool of eligible donors. It also increases ethnic diversity between donor and recipient populations, increasing the likelihood for alloimmunizations $[8,9]$. This problem is not evident in developing countries because their blood supply rarely meets existing needs. However, it is apparent in developed countries where, in spite of excess in blood supply for the general population, there is shortage of blood for minority patients harbouring blood group antigens that are rare among Caucasians.

In addition, availability of blood has allowed industrialized countries to implement aggressive surgical procedures and medical treatments for older individuals, creating a demographic revolution where for the first time the number of individuals $>60$ years of age is greater than that of those $<60$ years of age. Ageing of the population not only increases blood usage but also decreases the pool of eligible donors [10]. Because of the anticipated rate at which the age of the population will increase, it has been calculated that in industrialized countries the blood supply will no longer be adequate by 2050 [10].

\section{Cultured red blood cells as novel transfusion product}

For almost 50 years the transfusion medicine community has been searching for a safe blood substitute but without success. Recently, progress in culture techniques and proof-ofprinciple in animal models have allowed proposing red blood cells generated in culture (cRBCs) as a potential novel blood substitute [11]. Methods for cultivating small amounts of lineage-committed blood progenitor cells have been known for two decades [12] but massive production of cRBCs was made possible only recently by the discovery that dexamethasone and estradiol, the ligands for the glucocorticoid and oestrogen receptors, respectively, greatly increase the cell yield of erythroid cultures [13-16]. In theory, cultures 
seeded with stem cell sources currently discarded (low volume cord blood and buffy coats from adult blood donations) and stimulated with these two hormones in combination with haematopoietic growth factors may generate cRBCs in numbers sufficient for transfusion (at least $25 \times 10^{12} \mathrm{cRBCs}$ ) $[17,18]$. Proof-of-principle that $\mathrm{cRBCs}$ are functional in vivo was first proved by Dr. Nakamura and coworkers, who showed that cRBCs obtained from an immortalized embryonic stem cell (ES) line protect mice from lethal anaemia [19]. At first, this concept was considered unrealistic due to the complexity of the scale-up process and of its high costs. However, the amount of cRBCs required for phase I safety studies (the equivalent of 2-5 $\mathrm{ml}$ of blood) may be produced with current technologies at a reasonable cost. Comforted by this knowledge, Dr. Douay and coworkers defined good manufacturing practice (GMP) procedures to produce clinical grade cRBCs from G-CSF mobilized CD34 ${ }^{\text {pos }}$ cells of two volunteers [20]. These cRBCs were subjected to the extensive in-vitro and in-vivo characterization in animal models required prior to first-in-man evaluation of therapeutic products (http://www.clinicaltrials.gov/ct2/show/NCT00929266). 10 millions of these autologous cRBCs (the equivalent of $2 \mathrm{ml}$ of blood) were labelled by ${ }^{51} \mathrm{Cr}$ and transfused into the adult volunteer to measure in vivo survival. This study demonstrated that survival of cRBCs is comparable to that of native $\mathrm{RBC}$ and provided the first proof-ofprinciple in man for the use of ex vivo expanded RBC for transfusion [20]. Some of the investigators who are playing major roles in the development of cRBCs as transfusion products are depicted in Figure 1.

In spite of these progresses, scepticism, however, still exists fostered by the fact that nobody has still succeeded to produce $\mathrm{cRBCs}$ in numbers similar to those present in a unit of blood.

\section{Clinical applications for cRBCs feasible with current technologies}

In the current difficult economic climate, it is of paramount importance to envision and publicize meaningful clinical applications, diagnostic and/or therapeutic, pursuable with current technology to reassure the public of the vitality of the field and to secure the funding necessary to develop the technology necessary for scaled up production.

Diagnostic use of cRBCs as reagent RBCs may represent the first clinical application of this product. It is also possible that the studies necessary to validate this application may facilitate progression of cRBCs to clinical trial.

Reagent RBCs are obtained from highly selected donors with rare blood group phenotypes/ genotypes. These cells are used in diagnostic kits/devices used to identify alloimmunized individuals (both patients and blood donors) and to determine the specificity of the antibodies detected [21]. These assays can be performed with RBC from several $\mathrm{mL}$ of blood. However, periodic shortages of reagents RBC occur because rare ( 1 in $10^{5}$ donors) and very rare ( 1 in $10^{6}$ donors) donors may be either unavailable or temporarily/permanently deferred from donation. In addition, reagents RBCs for very-very rare ( 1 in $10^{7}$ donors) phenotypes are not commercially available. As only $\sim 2 \times 10^{8} \mathrm{RBCs}$ are sufficient to perform 100 of these assays and $10^{9}-10^{10} \mathrm{cRBCs}$ can be generated with current technology from the buffy coat of as little as $25 \mathrm{ml}$ of blood, it has been calculated that cRBCs obtained by 
culturing the buffy coat discarded from a rare blood donation $(500 \mathrm{ml})$ would provide sufficient amounts of cells for alloantibody identification for 1 year.

Blood centres have established programs for cryopreservation and international sharing of rare blood products for clinical use. The availability of molecular techniques for blood genotyping is allowing the establishment of national registries of blood donors with rare phenotypes and provides criteria for international recognition of these registries [22, 23]. However, storage procedures of buffy coats from rare to very-very rare blood donations are not implemented and international rules for sharing information and buffy coats from these donations have not been established. As a result, if a donor with rare to very-very rare phenotype becomes unable to donate, there is no mechanism to identify a suitable identical donor in another registry or to generate cRBCs from cryopreserved buffy coats. Given the limited numbers of donors with rare to very-very rare phenotypes available worldwide (no more than several hundreds), establishment of an international registry and GMP repository of buffy coats would be cost-effective. Generation of cRBCs from these cryopreserved buffy coats and validation of their use as reagent RBCs by evaluating their performance in currently accepted immunohematology assays is also not expensive and would provide a much needed diagnostic resource.

Confirmation of normal in vivo survival in autologous settings and determinations of in vivo cell survival and assessment of immunogenicity in allogenic settings represent clinical goals achievable with current technology. These issues may be addressed by small scale phase I trials in low risk autologous and allogenic situations in normal volunteers followed by low risk patient populations that require only local institutional review board approval but must be registered at clinicaltrials.gov. As it is unlikely that the G-CSF mobilization procedure used to obtain haematopoietic stem cells for the first-in-man study [20] will be routinely used to generate $\mathrm{cRBC}$ s for transfusion, the stem cell source to generate cRBCs for these trials will likely be represented by buffy coats from blood donations. Alloimmunized individuals currently donating blood as a source of antiserum for blood group antigen typing may represent a motivated normal population to volunteer for additional autologous and perhaps the first allogenic transfusion studies because they are occasionally challenged (where permitted) with small volumes (2-5 ml) of incompatible blood to boost antibody production (M von Lindern, personal communication). Comparison of the humoral and cellular immune responses of these alloimmunized individuals following transfusion of native and cRBCs from the same donor may provide important information about the immunogenicity of cRBCs. As alloimmunized individuals also contributed antisera necessary for validation of cRBCs as reagent red cells, data generated in the validation process may also provide additional safety data for these trials, reducing the cost of their regulatory submissions.

The low risk patients eligible for the first safety study should present clinical conditions in which transfusion of allogeneic cRBCs in numbers achievable with current techniques has therapeutic potential. As clinically significant increases in haemoglobin levels are achieved with doses of 5-10 $\mathrm{ml}$ of blood per $\mathrm{Kg}$ of body weight, infants, because of their small size, are the most logical candidates for this first study. Although considered a vulnerable population, the use of small children in clinical trials is not unusual. Safety and feasibility 
studies to assess the efficacy of cord blood for transfusion in small children with cerebral palsy or undergoing arterial switch operations in the first hours of life have been performed $[24,25]$. Alloimmunized children with sickle cell anaemia and patients with other haemoglobinopathies are those most likely to volunteer for these clinical studies because they are more prone to develop alloimmunization [11]. However, they may not be included in these initial studies because of their susceptibility to hyperhemolysis syndrome [26]. Because of their small size and immature immune system, pre-term neonates who undergo replacement transfusion for routine blood draws may represent the best population to evaluate in vivo survival and efficacy. The study may be designed as comparison of in vivo survival of comparable small volumes (2-5 ml each) of RBCs and cRBCs expanded from the same donor. Given the fact that the amounts of RBCs and cRBCs that will be transfused are comparable, continuous monitoring of blood values after these replacement procedures will also allow assessment of efficacy.

Children with Diamond Blackfan Anaemia (DBA) who require regular transfusions because they do not respond to glucocorticoids [27,28] may be considered for studies in patients with greater body mass. For reasons poorly understood the transfusion requirements of DBA patients increase over time and some of them also develop alloimmunization. As the spleen of DBA patients is not functional, these studies may not provide information on in vivo survival of cRBCs in the general population because cRBCs may persist for longer time in the presence of a compromised spleen. However, they will allow assessment of immunogenicity because, in contrast with pre-term infants, the immunological functions of DBA are normal. This study may be designed as comparison of in vivo survival of few $\mathrm{mL}$ $(2-10 \mathrm{ml})$ of cRBCs transfused concurrently with a similar volume of blood from the same donor. Techniques to label the two cell types with different tracers are available. Once this safety study is completed and prolonged survival of cRBCs in DBA patients demonstrated, an IND to conduct a second trial that will assess efficacy may be submitted and once approved, the trial may be conducted. We predict that this trial may be conducted with numbers of cRBCs that can be generated with current technology because the in vivo survival studies will have indicated that cRBCs survive for longer times in these patients.

\section{Barriers to the use of $\mathrm{CRBCs}$ as transfusion product in the general population}

Although the considerations outline above describe development of cRBCs as transfusion product for limited numbers of clinical applications, we believe that the results of these first trials will encourage the additional studies necessary to address the scientific and technical challenges that prevent today considering cRBCs as transfusion product for the general population. The major scientific and technical issues to be addressed are (1) identification of stem cell sources more suitable for generation of cRBCs and (2) scaled up production of cRBCs. 


\section{Genetically engineered stem cells as sources of cRBCs}

One of the most important aspects of cRBC production is the source of stem cells. The ideal stem cell source should be available as discarded material, have unlimited availability, be suitable to attain full erythroid maturation and not be immunogenic.

The cRBCs used for first-in-man administration were not derived from discarded material but from autologous CD34 ${ }^{\text {pos }}$ cells mobilized by G-CSF [20]. G-CSF mobilization is routinely performed to mobilize haematopoietic stem/progenitor cells for transplantation. Although it is similarly effective in individuals with Caucasian, African-american and Hispanic backgrounds [29], it may pose to donors risks unacceptable in the context of cRBC production. The leukoreduction byproducts (buffy coats) of adult blood donations are sources of haematopoietic stem/progenitor cells currently discarded that when cultured in media formulated with clinical grade components of human origin may theoretically generate cRBCs in numbers sufficient for 3-20 transfusions in adult recipients [30].

Cord blood is another source of haematopoietic stem/progenitor cells which is currently largely discarded because only a small percent of them (those $>90 \mathrm{ml}$ ) are collected for stem cell transplantation [31]. Each unit of ORh negative cord blood may theoretically generate 10-75 universal transfusion products [11,17], but as cord blood is presently characterized only for major blood group antigens, it may not be used to generate cRBCs for alloimmunized patients.

Current regulations recommend that the cell population used to initiate the process to produce a cell therapy product should be thoroughly characterized by means of specific biochemical, genetic, virologic, and bacteriologic tests. As these tests are expensive, cost consideration indicates that the ideal stem cell source should be capable to generate unlimited numbers of products. As both adult blood and cord blood may produce at most 50 red cell transfusion products each [11,17], the cost of their characterization adds significant burden to the cost of producing clinical grade cRBCs.

Embryonic stem cells (ES) and more recently, induced pluripotent stem cells (iPS) have been investigated as potential sources for the generation of universal ORh negative RBC. ES were shown to be capable of producing mature functional erythrocytes on a large scale [32, 33] andalthough ORh negative ES were not identified among the few lines originally available for research [34], it is predicted that thanks to the lift on the ban on ES research, suitable ES lines will be eventually identified among those that are being included in the NIH repository [35].

Lately, iPS obtained from human foetal and adult fibroblasts [36] and from neonatal fibroblasts [37] were used to produce RBC in vitro. iPS may be generated from any donor, including donors with rare blood phenotypes, representing a promising stem cell source to produce cRBCs for alloimmunized patients [22]. Another possibility allowed by advanced reprogramming technology is to reprogram any somatic cell directly into erythroblasts bypassing the pluripotent state by over-expression of suitable genes. A successful example of this approach is represented by the recent demonstration that over-expression of $\mathrm{p} 45 \mathrm{NF}$ E2/Maf turns human fibroblasts into megakaryocytes [38]. 
Large scale generation of cRBCs directly from Es, iPS or from reprogrammed fibroblasts will be as costly and time-consuming as that to produce them from cord blood or adult blood. The population of self-renewing erythroblasts recently discovered in early mouse embryos [39] suggests that it may be possible, by over-expressing a combination of suitable genes, to immortalize human erythroblasts obtained in culture into cell line-like cells capable of extensive proliferation but conserving their ability to differentiate into cRBCs. Ideally, the proliferation of these cell lines would require only erythropoietin and steroids, relatively inexpensive hormones, and differentiation would be induced by erythropoietin alone. A repertoire of erythroblast-derived cell lines expressing combinations of rare blood phenotypes would then represent the ultimate unlimited cell source for inexpensive production of cRBCs.

There is a tendency of immortalized cells to acquire karyotypic abnormalities but this caveat should not represent a problem as cRBCs do not contain genetic material. In addition, the leukoreduction plus radiation procedures developed to prevent alloimmunization to HLA antigens present on leukocytes and graft versus host disease after transfusion [8], may assure that cRBCs are completely devoid of cells capable of proliferation.

\section{Scaling up issues of the $\mathrm{CRBC}$ production process}

Generating large amounts of cRBCs at a reasonable cost is the most challenging technical obstacle to make transfusion with these cells a reality. Erythroid cells do not proliferate at a concentration $>10^{6}$ cells $/ \mathrm{ml}$. Therefore, production of cRBCs in numbers sufficient for a transfusion $\left(2 \times 10^{12} \mathrm{RBCs}\right)$ requires at least $25 \times 10^{3} 1$ of culture media [18]. The only equipments that may manage such large volumes are the bioreactors developed by the biotechnology industry such as the WAWE System or perfusion hollow fibres [40,41]. Whether one of these systems is suitable to produce cRBCs and would allow them to grow at greater concentrations, decreasing production costs, is currently unknown but it is an interesting proposition. Additional reductions in production costs may be expected by rationalization of the criteria to characterize the stem cell source and of the use of growth factors to stimulate the culture and by identification of cheaper culture components.

\section{Acknowledgments}

This study was supported by grants from the Italian Centro Nazionale Sangue, Rome, Italy and from NHLBI (HL116329-01), Bethesda, MD, USA

\section{References}

1. Alter HJ, Klein HG. The hazards of blood transfusion in historical perspective. Blood. 2008; 112:2617. [PubMed: 18809775]

2. Services, T.U.D.o H.a H.. , editor. The 2009 National Blood Collection and Utilization Survey Report. US Department of Health and Human Services, Office of the Assistant Secretary for Health; Washington, District of Columbia: 2011.

3. van der Poel, CL.; Janssen, MP.; Behr-Gross, ME. The Collection, Testing and Use of Blood and Blood Components in Europe. 2008. Report. http:/www.edqm.eu/medias/fichiers/ The_Collection_Testing_and_Use_of_Blood_and_Blood_3.pdf

4. Nightingale S, Wanamaker V, Silverman B, et al. Use of sentinel sites for daily monitoring of the US blood supply. Transfusion. 2003; 43:364. [PubMed: 12675723] 
5. Sullivan MT, Cotten R, Read EJ, et al. Blood collection and transfusion in the United States in 2001. Transfusion. 2007; 47:385. [PubMed: 17319817]

6. Epstein JS, Holmberg JA. Progress in monitoring blood safety. Transfusion. 2010; 50:1408. [PubMed: 20636529]

7. WHO. Global Blood Safety and Availability. Key facts and figures. 2010. http://www.who.int/ mediacentre/factsheets/fs279/en/index.html

8. Zimring JC, Welniak L, Semple JW, et al. Current problems and future directions of transfusioninduced alloimmunization: summary of an NHLBI working group. Transfusion. 2011; 51:435. [PubMed: 21251006]

9. Vichinsky EP, Earles A, Johnson RA, et al. Alloimmunization in sickle cell anemia and transfusion of racially unmatched blood. N Engl J Med. 1990; 322:1617. [PubMed: 2342522]

10. Ali A, Auvinen MK, Rautonen J. The aging population poses a global challenge for blood services. Transfusion. 2010; 50:584. [PubMed: 19912582]

11. Migliaccio AR, Whitsett C, Papayannopoulou T, et al. The potential of stem cells as an in vitro source of red blood cells for transfusion. Cell Stem Cell. 2012; 10:115. [PubMed: 22305561]

12. Papayannopoulou, Th; Abkowitz, J.; D’Andrea, A., et al. Biology of erythropoiesis, erythroid differentiation and maturation. In: Hoffman, R.; Benz, EJ.; Shattil, SJ.; Furie, B.; Cohen, HJ.; Silberstein, LE.; McGlave, P.; Heslop, H., editors. Hematology: Basic Principles and Practice. 5. Philadelphia, PA, USA: Elsevier; 2009. p. 276-299.

13. Fibach E, Manor D, Oppenheim A, et al. Proliferation and maturation of human erythroid progenitors in liquid culture. Blood. 1989; 73:100. [PubMed: 2910352]

14. Panzenbock B, Bartunek P, Mapara MY, et al. Growth and differentiation of human stem cell factor/erythropoietin-dependent erythroid progenitor cells in vitro. Blood. 1998; 92:3658. [PubMed: 9808559]

15. von Lindern M, Zauner W, Mellitzer G, et al. The glucocorticoid receptor cooperates with the erythropoietin receptor and c-Kit to enhance and sustain proliferation of erythroid progenitors in vitro. Blood. 1999; 94:550. [PubMed: 10397722]

16. Migliaccio G, Di Pietro R, di Giacomo V, et al. In vitro mass production of human erythroid cells from the blood of normal donors and of thalassemic patients. Blood Cells Mol Dis. 2002; 28:169. [PubMed: 12064913]

17. Peyrard T, Bardiaux L, Krause C, et al. Banking of pluripotent adult stem cells as an unlimited source for red blood cell production: potential applications for alloimmunized patients and rare blood challenges. Transfus Med Rev. 2011; 25:206. [PubMed: 21377319]

18. Migliaccio AR, Whitsett C, Migliaccio G. Erythroid cells in vitro: from developmental biology to blood transfusion products. Curr Opin Hematol. 2009; 16:259. [PubMed: 19444099]

19. Hiroyama T, Miharada K, Sudo K, et al. Establishment of mouse embryonic stem cell-derived erythroid progenitor cell lines able to produce functional red blood cells. PLoS ONE. 2008; 3:e1544. [PubMed: 18253492]

20. Giarratana MC, Rouard H, Dumont A, et al. Proof of principle for transfusion of in vitro-generated red blood cells. Blood. 2011; 118:5071. [PubMed: 21885599]

21. Castro O, Sandler SG, Houston-Yu P, et al. Predicting the effect of transfusing only phenotypematched RBCs to patients with sickle cell disease: theoretical and practical implications. Transfusion. 2002; 42:684. [PubMed: 12147019]

22. Lapillonne H, Kobari L, Mazurier C, et al. Red blood cell generation from human induced pluripotent stem cells: perspectives for transfusion medicine. Haematologica. 2010; 95:1651. [PubMed: 20494935]

23. Rivelli N, Villa MA, Paccapelo C, et al. The Lombardy Rare Donor Program. Transfusion. 2013:1-7. [Epub ahead of print]. 10.2450/2013.0182-12

24. Chasovskyi K, Fedevych O, Vorobiova G, et al. Arterial Switch Operation in the First Hours of Life Using Autologous Umbilical Cord Blood. Ann Thorac Surg. 2012; 93:1571. [PubMed: 22459547]

25. Papadopoulos KI, Low SS, Aw TC, et al. Safety and feasibility of autologous umbilical cord blood transfusion in 2 toddlers with cerebral palsy and the role of low dose granulocyte-colony stimulating factor injections. Restor Neurol Neurosci. 2011; 29:17. [PubMed: 21335665] 
26. Win N, New H, Lee E, et al. Hyperhemolysis syndrome in sickle cell disease: case report (recurrent episode) and literature review. Transfusion. 2008; 48:1231. [PubMed: 18373500]

27. Ellis SR, Lipton JM. Diamond Blackfan anemia: a disorder of red blood cell development. Curr Top Dev Biol. 2008; 82:217. [PubMed: 18282522]

28. Vlachos A, Ball S, Dahl N, et al. Diagnosing and treating Diamond Blackfan anaemia: results of an international clinical consensus conference. Br J Haematol. 2008; 142:859. [PubMed: 18671700]

29. Carilli AR, Sugrue MW, Rosenau EH, et al. African American adult apheresis donors respond to granulocyte-colony-stimulating factor with neutrophil and progenitor cell yields comparable to those of Caucasian and Hispanic donors. Transfusion. 2012; 52:166. [PubMed: 21790625]

30. Migliaccio G, Sanchez M, Masiello F, et al. Humanized culture medium for clinical expansion of human erythroblasts. Cell Transplant. 2010; 19:453. [PubMed: 20149301]

31. Broxmeyer HE. Umbilical cord transplantation: epilogue. Semin Hematol. 2010; 47:97. [PubMed: 20109617]

32. Lu SJ, Feng Q, Park JS, et al. Biologic properties and enucleation of red blood cells from human embryonic stem cells. Blood. 2008; 112:4475. [PubMed: 18713948]

33. Olivier EN, Qiu C, Velho M, et al. Large-scale production of embryonic red blood cells from human embryonic stem cells. Exp Hematol. 2006; 34:1635. [PubMed: 17157159]

34. Bonig H, Chang KH, Geisen C, et al. Blood types of current embryonic stem cell lines are not conducive to culturing "universal-donor" red blood cells. Transfusion. 2008; 48:1039. [PubMed: 18454742]

35. US National Health Institute. Stem Cell Registry. http://stem-cells.nih.gov/research/registry

36. Chang KH, Huang A, Hirata RK, et al. Globin phenotype of erythroid cells derived from human induced pluripotent stem cells. Blood. 2010; 115:2553. [PubMed: 20339107]

37. Chang CJ, Mitra K, Koya M, et al. Production of embryonic and fetal-like red blood cells from human induced pluripotent stem cells. PLoS ONE. 2011; 6:e25761. [PubMed: 22022444]

38. Wang Y, Ono Y, Ikeda Y, et al. Induction of Megakaryocytes From Fibroblasts by p45NF-E2/Maf. Blood. 2011; 11:908a.

39. England SJ, McGrath KE, Frame JM, et al. Immature erythroblasts with extensive ex vivo selfrenewal capacity emerge from the early mammalian fetus. Blood. 2011; 117:2708. [PubMed: 21127173]

40. Timmins NE, Athanasas S, Gunther M, et al. Ultra-high-yield manufacture of red blood cells from hematopoietic stem cells. Tissue Eng Part C Methods. 2011; 17:1131. [PubMed: 21736478]

41. Housler GJ, Miki T, Schmelzer E, et al. Compartmental Hollow Fiber Capillary Membrane Based Bioreactor Technology for in vitro Studies on Red Blood Cell Lineage Direction of Hematopoietic Stem Cells. Tissue Eng Part C Methods. 2012; 18:133. [PubMed: 21933020] 

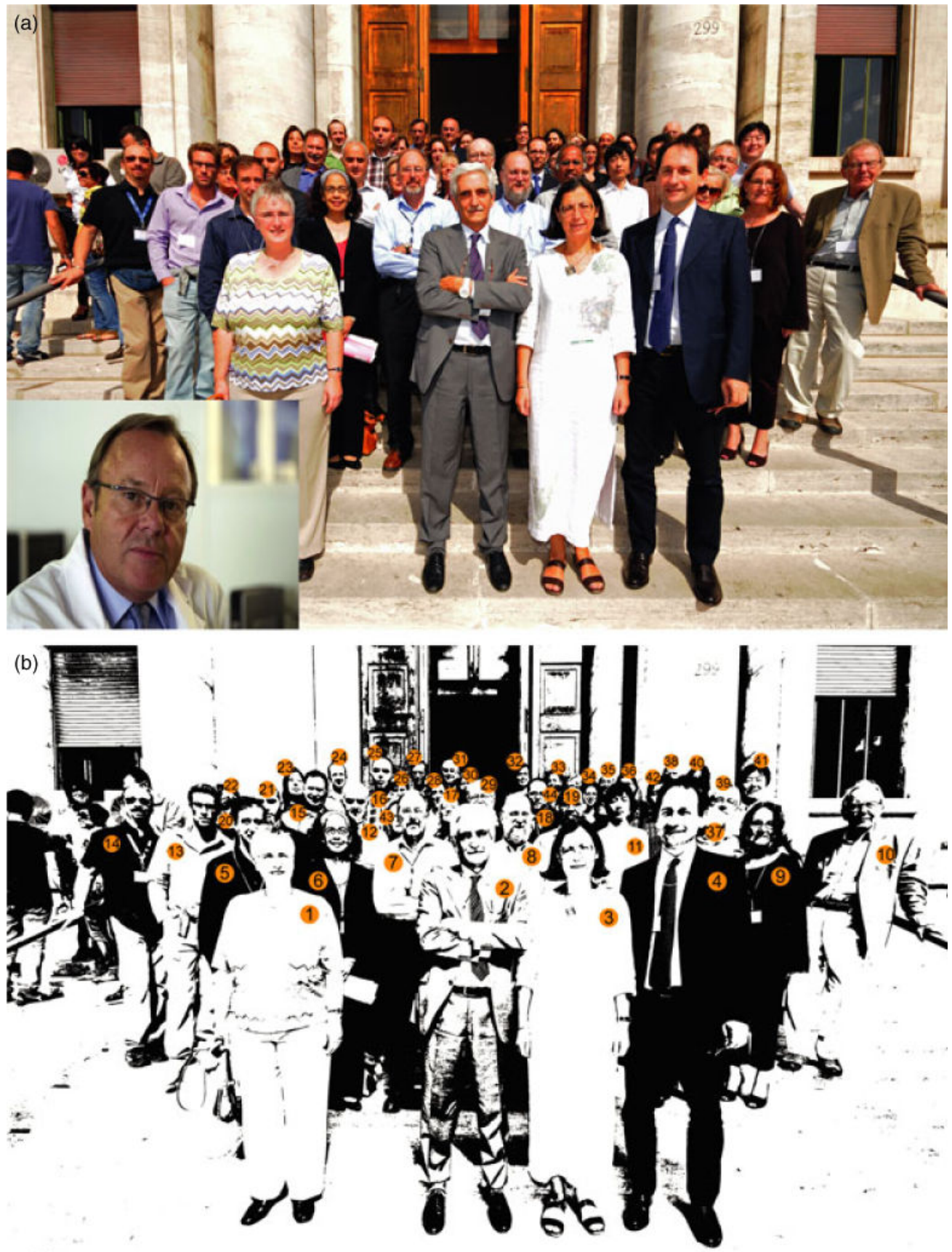

Fig. 1.

(a) Participants of the workshop Making blood from progenitors ex vivo held in Rome on September 19th, 2011 on the background of the Italian National Institute of Health (Istituto Superiore di Sanità, ISS) organized by Drs. Anna Rita Migliaccio, Giuliano Grazzini, head of the National Blood Center and Ruggero De Maria, head of the Department of Hematology, Oncology and Molecular Medicine at the ISS. This meeting included most of the scientists who are playing a major role in the development of this field to discuss the scientific and logistic barriers to be overcome for a successful translation of this innovative 
into clinical practice. (b) Numerical list of participants: (1) Marieke von Lindern, (2) Giuliano Grazzini, (3) Anna Rita Migliaccio, (4) Ruggero De Maria, (5) John Crispino, (6) Carolyn Whitsett, (7) David Anstee, (8) James Palis, (9) Kateri Moore, (10) George Stamatoyannopoulou, (11) Hirohama Takahashi, (12) Godfrey Grech, (13) Claudio Zolla, (14) Fabrizio Martelli, (15) Lello Zolla, (16) Emile Van Den Akker, (17) Vanda Randi, (18) Francesco Alviano, (19) Mohandas Narla, (20) Laura Forte, (21) Alessandro DAmico, (22) Alexandra Gampel, (23) Anna Concetta Berardi, (24) Ann Zeuner, (25) Claudio Velati, (26) Elena Masselli, (27) Lilian Varricchio, (28) Barbara Ghinassi and Valentina Tirelli, (29) Massimo Sanchez, (30) Cintia Carella, (31) Thalia Papayannopoulou, (32) Ihor Lemischka, (33) Maria Verrucci, (34) Yukio Nakamura, (35) Laura Salvaneschi, (36) Clara

Camaschella. The name of scientists most involved in this research is indicated in bold. The photograph of Prof L. Douay is presented in the insert. 